\title{
Comparative Evaluation of Palpable Breast Lumps by Digital Breast Tomosynthesis and MRI Breast with its Histopathological Correlation
}

\author{
Sangeeta Saxena ${ }^{1}$, Dharm Raj Meena ${ }^{2}$, Tusharika Sharma ${ }^{3}$, Harshvardhan Khokhar ${ }^{4}$ \\ ${ }^{1}$ Senior Professor, Department of Radiodiagnosis, Government Medical College and Associated Group of Hospitals, Kota \\ 324001, ${ }^{2}$ Professor and Head, Department of Radiodiagnosis, Government Medical College and Associated Group of \\ Hospitals, Kota 324001, ${ }^{3}$ Resident, Department of Radiodiagnosis, Government Medical College and Associated Group of \\ Hospitals, Kota 324001, ${ }^{4}$ Assistant Professor, Department of Radiodiagnosis, Government Medical College and Associated \\ Group of Hospitals, Kota 324001, India
}

Corresponding author: Dr. Tusharika Sharma, Resident, Department of Radiodiagnosis, Govt. Medical College and Associated Group of Hospitals, Kota 324001, India

DOI: http://dx.doi.org/10.21276/ijcmsr.2019.4.2.29

How to cite this article: Sangeeta Saxena, Dharm Raj Meena, Tusharika Sharma, Harshvardhan Khokhar. Comparative evaluation of palpable breast lumps by digital breast tomosynthesis and mri breast with its histopathological correlation. International Journal of Contemporary Medicine Surgery and Radiology. 2019;4(2):B132-B138.

\section{A B S T R A C T}

Introduction: Breast cancer is the leading cause of mortality and morbidity in females worldwide. Combating this, needs early detection of malignancy and prompt management. Various modalities available are - digital mammography +/tomosynthesis (DBT), ultrasound with colour Doppler and elastography, dynamic contrast enhanced MRI

(DCE-MRI) with MRI being considered as the best. This study was done to compare efficacy of digital breast tomosynthesis and MRI breast in evaluation of palpable breast masses and other suspicious breast findings.

Material and methods: A prospective study of 50 patients was carried out in the Department of Radio-diagnosis, Government Medical College, Kota [Rajasthan]. Females (20-72 years) with clinically palpable breast lump/nipple erosion/ nipple retraction/nipple discharge / for screening purposes / BI-RADS 3,4,5 were included. Cases on chemotherapy/ radiotherapy /post operative cases/recent biopsy done from the lesion/painful breast lesions/any contraindication to MRI /pregnant females/ BIRADS 1,2,6 were excluded. DBT and MRI was performed in each patient and results were correlated with histopathology.

Results: Well circumscribed margins was most commonly associated with benignity Spiculated margins and gross architectural distortion(seen on both DBT and MRI), fine pleomorphic/grouped/linear/segmental/amorphous calcifications (seen on DBT), rim enhancement of mass with kinetic curve III, clumped/linear/segmental/focal non mass like enhancement (coinciding with calcifications on DBT), adjacent invasion and metastasis were associated with malignancy.

Conclusion:Differentiation between benign and malignant lesions by DBT had $77.2 \%$ specificity with DCE- MRI having 95.45\% specificity. Difference was statistically significant (Chi-square $=29.429$ with 1 degree of freedom; $P<0.001 S$ ).

Keywords: Breast, Imaging, Magnetic Resonance Imaging, Tomosynthesis

\section{INTRODUCTION}

Patients complaining of breast disorders form a large proportion of outpatients in general surgical clinics. The presence of a lump in the breast is a great cause of anxiety and apprehension in females, young or old. Many patients with complaints of breast disorders have cyclical mastalgia, nodularity or asymmetry, but a good proportion indeed present with breast lumps. Of these lumps, the most crucial diagnosis is that of breast malignancy. Breast masses have two main types of etiologies -benign or malignant. Fibroadenoma is the most common benign breast mass; invasive ductal carcinoma is the most common malignancy. Although most masses are benign but incidence of breast cancer has been increasing in the past years especially in younger age groups thus making breast malignancy a national and global health burden

- Current scenario according to GLOBOCAN 2018 shows that among females worldwide, breast cancer is the most commonly diagnosed cancer and the leading cause of cancer death

- GLOBOCAN 2018: INDIA FACTSHEET shows a similar picture of breast cancer being the most common cancer (incidence) and the most common cause of cancer deaths (mortality) in females respectively

- Although most breast cancers occur in women older than 50 years but recent trends show an increase in incidence among younger females (less than 40 years). ${ }^{1}$

However, according to "cancer statistics,2018" published in 
India has a low survival rate (66\%) possibly due to lack of awareness, ignorance leading to patients of carcinoma breast presenting in late stages of disease- according to the study titled Global surveillance of trends in cancer survival 200014 (CONCORD-3). ${ }^{2}$

This emphasises the need of rampant awareness,rigorous efforts for an early, accurate diagnosis and management of breast malignancy which is why the aim of breast imaging is to assess probability of a lesion being benign or malignant using multimodality approach of digital mammography+/tomosynthesis, ultrasound with colour Doppler,elastography and dynamic contrast enhanced MRI.

This study was done to compare efficacy of digital breast tomosynthesis and MRI breast in evaluation of palpable breast masses and other suspicious breast findings.

\section{MATERIAL AND METHODS}

A prospective study was conducted in the Department of Radio diagnosis, Government Medical College Kota, Rajasthan. We included 50 patients after proper informed and written consent during the period of study. Patients were 20-72 years of age range. In all patients, clinical complaints and relevant demographic data was recorded. Each patient underwent breast tomosynthesis and dynamic contrast enhanced MRI breast and results were correlated with histopathology.

Inclusion criteria: Patients with clinically palpable breast lump/nipple retraction/erosion/ nipple discharge /for screening mammography/BI-RADS 3,4,5.

Exclusion criteria: BI-RADS 1,2,6/prior surgery/recent biopsy done from lesion/on chemotherapy/radiotherapy/ painful breast lesion/pregnant females/any contraindication to MRI.

In all patients, Digital Breast Tomosynthesis was done using Alpinion Acheiva 15. Cranio-caudal (CC) and mediolateraloblique (MLO) views of bilateral breasts were taken in 3D using tomosynthesis and then $2 \mathrm{D}$ images were synthesized from them and interpretation was done using ACR BIRADS lexicon and lesions were assigned a preliminary BIRADS category.Further dynamic contrast enhanced MRI on 1.5 T Phillips Achieva machine was performed using dedicated 16 channel breast coil (patient position prone). MR - protocol followed was-

- T2-weighted axial fat suppressed

- T1-weighted axial non-fat-suppressed 3D FSPGR

- T1-weighted fat suppressed axial3D FSPGR before and following contrast administration (Dynamic Contrast Imaging)

- 2-mm slice thickness, no gap

- Subtraction images

- Computer aided detection

Evaluation was done using ACR MRI BIRADS lexicon and appropriate final BI-RADS category was assigned to the lesion and results were correlated with histopathology.

\section{STATISTICAL ANALYSIS}

Sensitivity, specificity, positive and negative predictive value and diagnostic accuracy for digital breast tomosynthesis and MR mammography was calculated using Mc-Nemar test. P values were calculated using Chi square test.

- In our study sensitivity, specificity, PPV, NPV and diagnostic accuracy of DBT against histopathology was $100 \%, 77.2 \%, 84.85 \%, 100 \%$ and $90.0 \%$ respectively

- In our study, sensitivity, specificity, PPV, NPV and diagnostic accuracy of MRI against histopathology was 100\%, 95.45\%, 96.55\%,100\% and 98\% respectively

- Comparison of specificity of MR mammography and digital breast tomosynthesis - Difference was statistically significant (Chi-square $=29.429$ with 1 degree of freedom; P <0.001S).

\section{RESULTS}

Out of 50 cases taken, on histopathology 22 cases were benign while 28 cases were proven malignant with the most common benign and malignant lesion being fibroadenoma and ductal carcinoma respectively (Table-1). ACR BIRADS lexicon was used to define the lesions on DBT and mammography (Table-2,3).

On tomosynthesis and DCE- MRI, all fibroadenomas were seen as oval shaped lesions with well circumscribed margins. Malignancy in our study had three major presentations -

1. Mass lesion

2. Architectural distortion/asymmetry.

3. Suspicious morphology calcification on DBT (which coincided with suspicious NMLE on MRI) without the presence of any mass or architectural distortion

The margins of the mass lesion (irrespective of any associated calcification) was the most important criteria for differentiating benign and malignant lesions with spiculated and indistinct margins being the ones commonly associated with malignancy. Irregular shape was most commonly seen associated with malignancy. Well circumscribed margins

\begin{tabular}{|l|l|c|c|}
\hline Histopathological diagnosis & Number of patients & Percentage \\
\hline \multirow{4}{*}{ Benign } & Fibroadenoma & 17 & $34 \%$ \\
\cline { 2 - 4 } & Granulomatous mastitis & 02 & $4 \%$ \\
\cline { 2 - 4 } & Fat necrosis & 02 & $4 \%$ \\
\cline { 2 - 4 } & Chronic mastitis & 01 & $2 \%$ \\
\hline \multirow{4}{*}{ Malignant } & Ductal carcinoma & 19 & $38 \%$ \\
\cline { 2 - 4 } & Pagets disease & 02 & $4 \%$ \\
\cline { 2 - 4 } & Lobular carcinoma & 04 & $8 \%$ \\
\cline { 2 - 4 } & DCIS & 03 & $6 \%$ \\
\hline Total & & 50 & 100 \\
\hline
\end{tabular}




\begin{tabular}{|c|c|c|}
\hline Findings & Benign findings on DBT & Suspicious findings on DBT \\
\hline \multirow[t]{2}{*}{ Shape } & Round shape & \multirow[t]{2}{*}{ Irregular shape } \\
\hline & Oval shape & \\
\hline \multirow[t]{3}{*}{ Margins } & \multirow[t]{3}{*}{ Well circumscribed } & Microlobulated margins \\
\hline & & Indistinct margins \\
\hline & & Spiculated margins \\
\hline \multirow[t]{2}{*}{ Microcalcification } & Skin/vascular/rim/popcorn like like calcification & $\begin{array}{l}\text { Linear/linearbranching/fine pleomorphic/amorphous } \\
\text { calcification }\end{array}$ \\
\hline & $\begin{array}{l}\text { Bilateral diffuse/ multiregional Distribution of micro- } \\
\text { calcification }\end{array}$ & $\begin{array}{l}\text { Linear/ ductal / segmental/ grouped /single regional } \\
\text { distribution pattern of microcalcification }\end{array}$ \\
\hline \multirow[t]{5}{*}{ Other findings } & & Nipple Erosion /retraction \\
\hline & & Skin thickening/retraction \\
\hline & & Global/focal asymmetry \\
\hline & & Architectural distortion \\
\hline & & Involvement of pectoralis muscle \\
\hline
\end{tabular}

\begin{tabular}{|l|l|l|}
\hline Findings & Less suspicious features on DCE -MRI & Major suspicious features on DCE-MRI \\
\hline \multirow{2}{*}{ Shape } & Oval shape & Irregular shape \\
\cline { 2 - 2 } & Round shape & Irregular/spiculated margins \\
\hline Margins & Well circumscribed margins & Heterogenous/Rim enhancement \\
\hline Internal enhancement & Homogenous & Type II / III \\
\hline Kinetic curve & Type I & Low to intermediate \\
\hline T2 Signal intensity & High & $\begin{array}{l}\text { Segmental/clumped/linear/regional/ Focal/clustered } \\
\text { ring NMLE }\end{array}$ \\
\hline Non mass like enhancement & Bilateral diffuse/multiregional NMLE \\
\cline { 2 - 2 } & \multicolumn{2}{|c|}{ Table-3: Analysis of MR breast ACR- BIRADS lexicon in our study } \\
\hline \multicolumn{2}{|l}{}
\end{tabular}

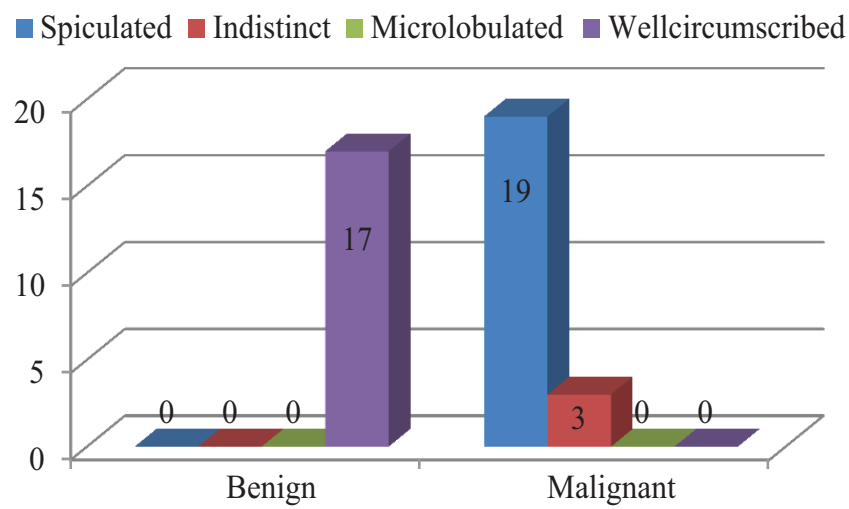

Figure-1: Bar graph showing assessment of mass margins in our study
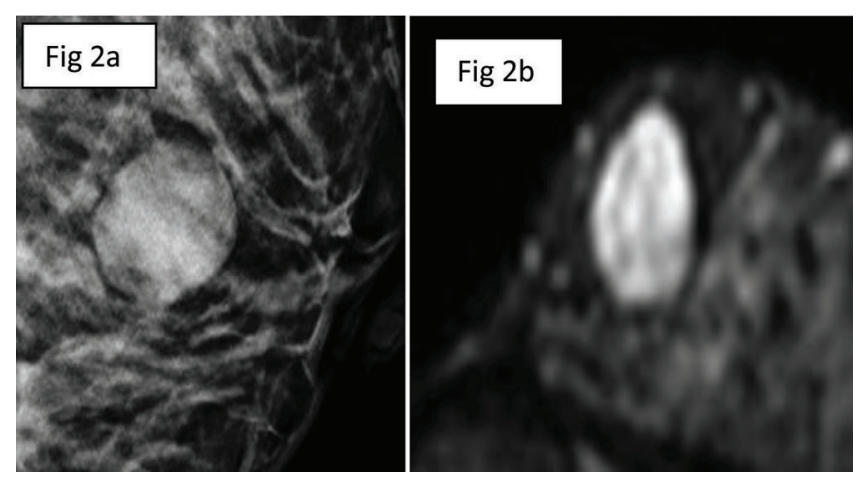

Figure-2: (a) shows oval shaped high density lesion with well circumscribed margins on DBT; (b) oval shaped well circumscribed lesion with homogenous enhancement; HPEFibroadenoma
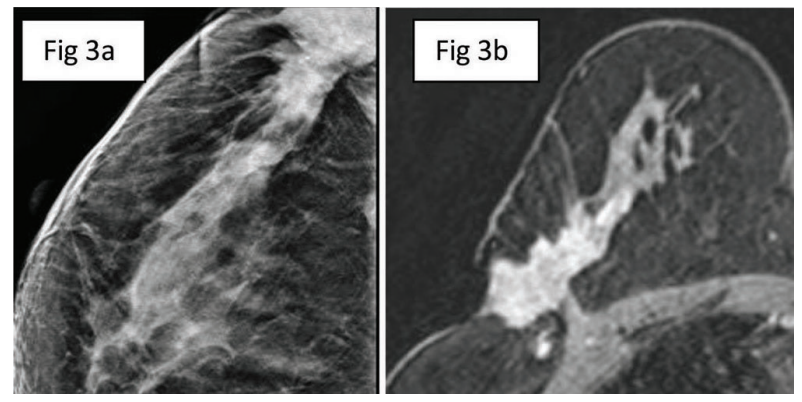

Figure-3: (a) Right breast high density irregular mass with spiculated margins and amorphous calcifications; (b) Dynamic contrast enhanced MRI shows enhancing mass with spiculated margins and infilterating overlying skin causing pectoralis invasion on right side (hook sign); HPE - Infilterating lobular carcinoma
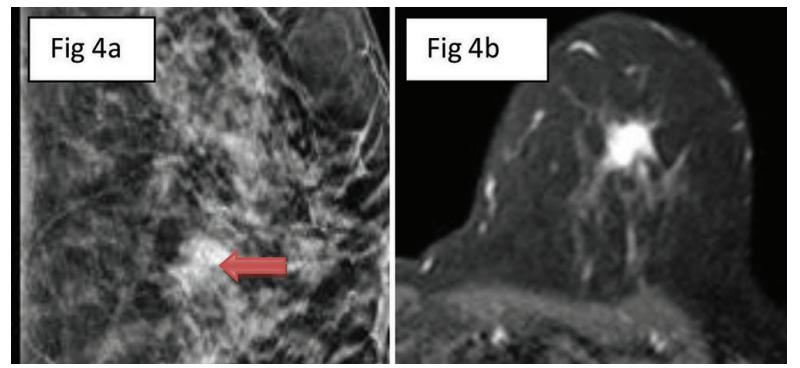

Figure-4: (a) Left breast craniocaudal view shows irregular high density mass lesion with spiculated margins; (b) Dynamic contrast enhanced MR solid homogenously enhancing mass lesion with spiculated margins; HPE Ductal carcinoma 
- Progressive/curve I a Plateau/curve II

Washout/curve III

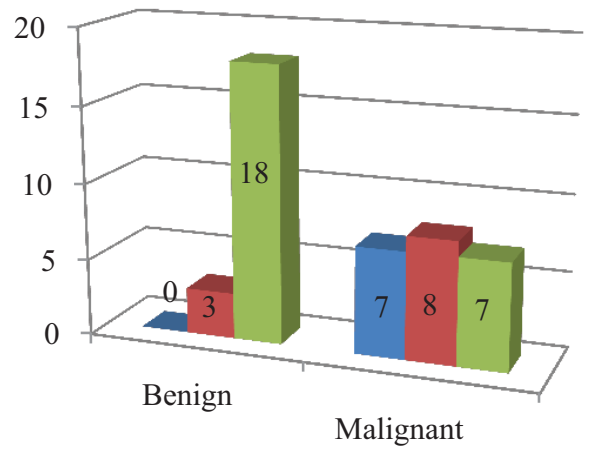

Figure-5: Kinetic curve assessment in our study

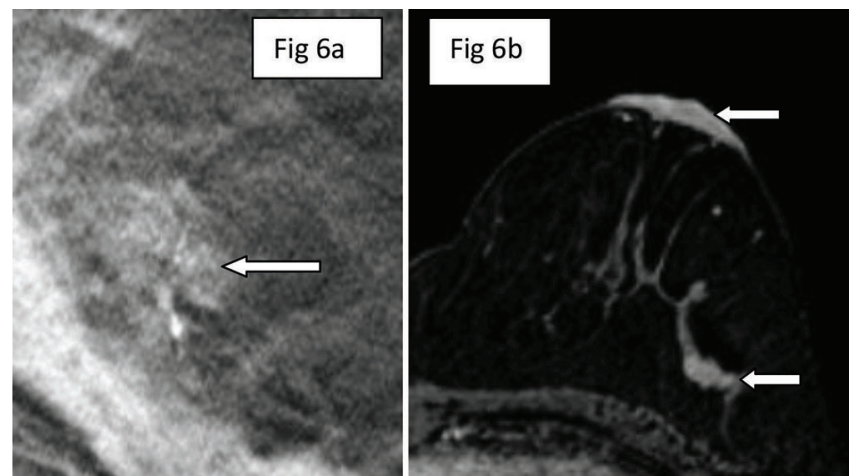

Figure-6: (a) DBT (magnified view) shows an area of fine pleomorphic calcification in grouped distribution with and associated architectural distortion; (b) Nipple erosion (with enhancement) and linear non mass like enhancement on dynamic contrast enhanced MRI coinciding with the calcification on DBT; HPE - Pagets disease with synchronous DCIS
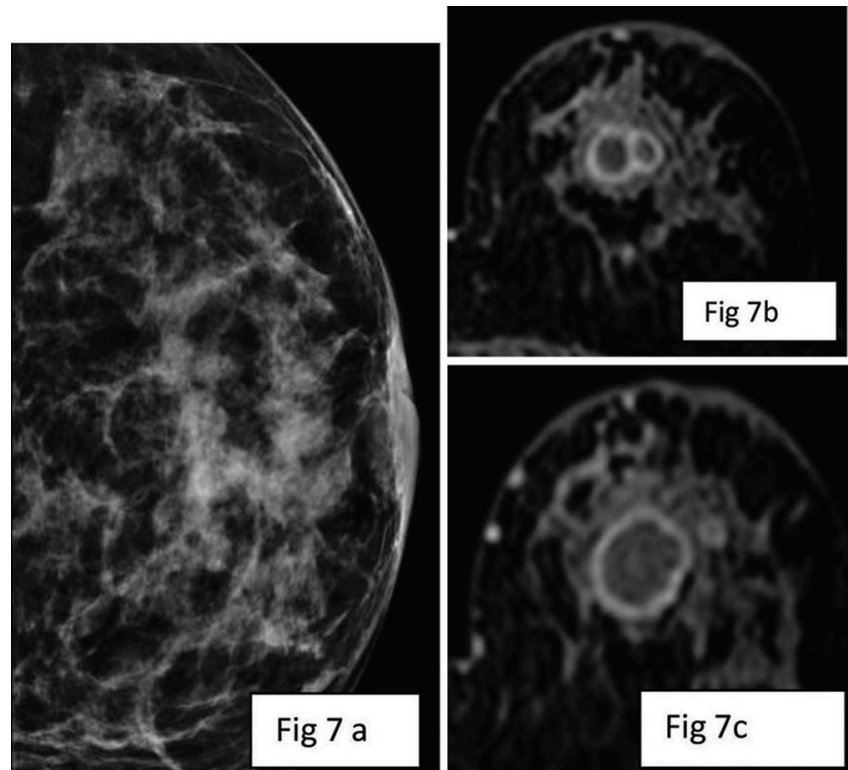

Figure-7: (a) DBT shows architectural distortion seen involving left upper outer quadrant with nipple retraction and thickened nipple - areola complex; (b and c) Multiple peripherally enhancing abscesses seen in dynamic contrast enhanced MRI; HPE - Granulomatous mastitis were $100 \%$ specific for benignity (Figure 1).

\section{Malignancy either presented as isolated calcifications (fine pleomorphic, grouped/linear/ amorphous)}

1. Without any coexisting mass lesion/associated architectural distortion - 02 cases of DCIS

2. With focal asymmetry and nipple erosion -02 cases of Pagets disease

These calcifications coincided with suspicious NMLE on DCE-MRI-focal/linear, clumped/heterogenous NMLE.

Nine cases (05 benign and 04 malignant) presented as architectural distortion/asymmetry in our study and tomosynthesis was unable to differentiate between benign and malignant etiology. An adjuvant second look ultrasound or DCE-MRI was able to differentiate between the two.

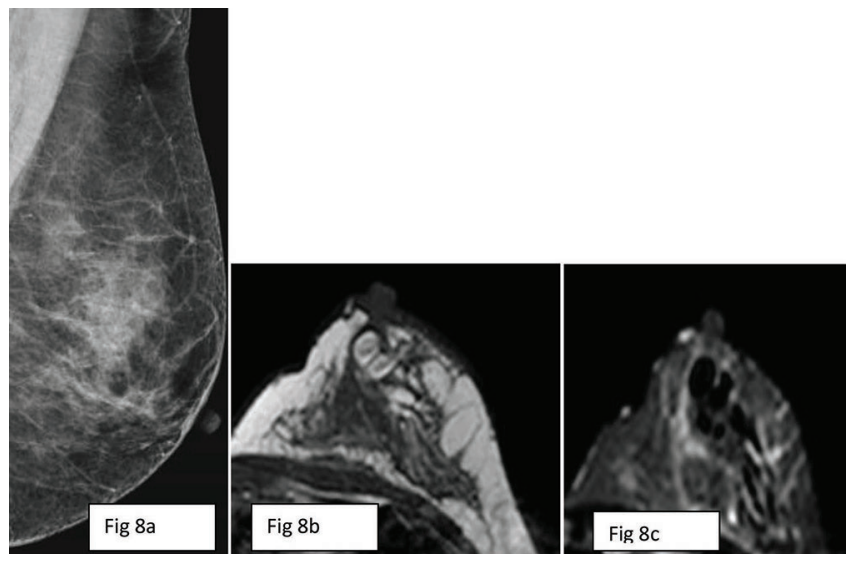

Figure-8: (a) DBT shows area of asymmetry/architectural distortion involving left upper breast; (b and c) multiple fat globules are seen which appear hyperintense on T1W; (b) with suppression of signal intensity and peripheral enhancement, perilesional inflammation on dynamic contrast enhanced MRI; HPE - Fat necrosis

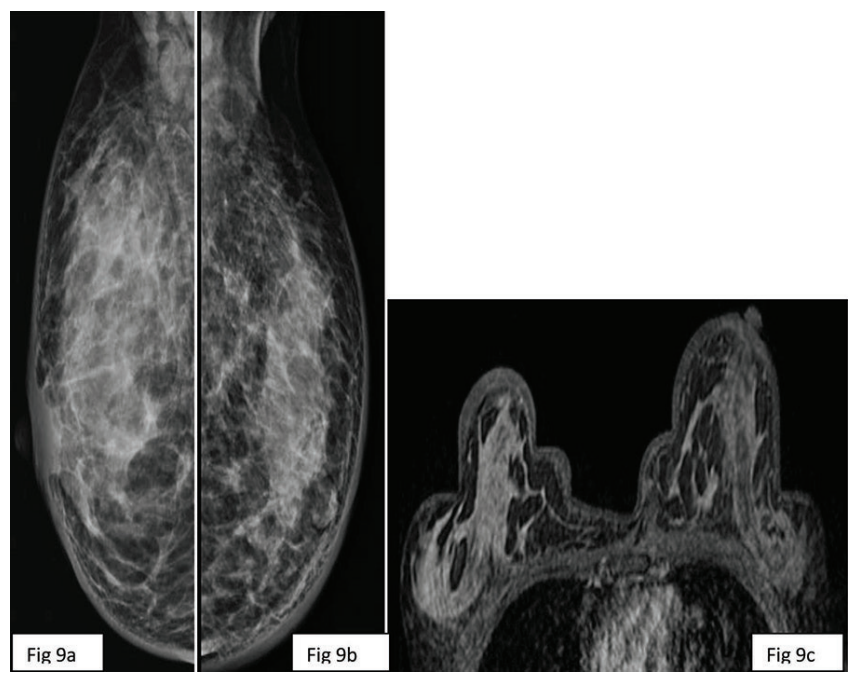

Figure-9: (a and b) Bilateral breast craniocaudal and mediolateral oblique views show bulky breasts with significant skin thickening and internal architectural distortion; (c) bilateral variable diffuse non mass like enhancement with skin thickening on dynamic contrast enhanced MRI; HPE Bilateral infilterating ductal carcinoma 


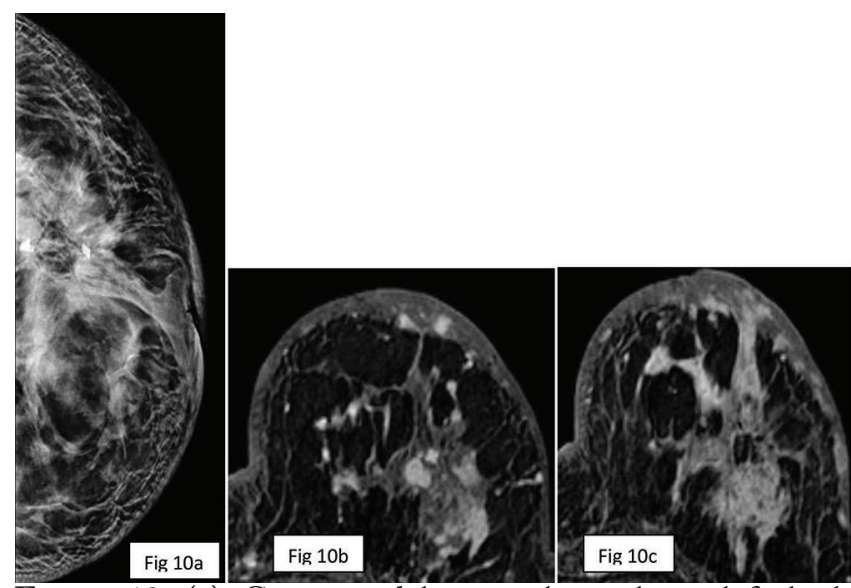

Figure-10: (a) Craniocaudal view shows large left high density mass lesion with spiculated margins and surrounding architectural distortion and skin thickening; (b and c) Dynamic contrast enhanced MRI showing enhancing mass lesion with spiculated margins and multiple satellite lesions (multifocal and multicentric) with infilteration seen extending into the retracted nipple with skin thickening (multiple enhancing nodular skin deposits); HPE- Invasive breast cancer micropapillary type

\section{DISCUSSION}

Our study consisted of 50 patients and we used ACR BIRADS lexicon

(mammography and MR) for describing the lesion.In our study we were able to correctly diagnose breast masses(benign or malignant) based on the margins (98\%) regardless of their histological variability. Benign mass lesions were oval in shape with well circumscribed margins and variable pattern of enhancement depending on the underlying cause (homogenous enhancement in fibroadenoma, peripheral enhancement in multiple abscesses of granulomatous mastitis, peripheral enhancement of fat globules in fat necrosis). Malignancy showed spiculated margins or irregular margins. None of the benign lesions showed spiculated margins (Figure 2,3,4). Enhancement pattern seen in malignant masses was as follows-heterogenous(68.1\%), homogenous $(22.7 \%)$, peripheral (9\%). Irregular shape was the most common shape seen in the malignant masses in our study(95\%).

Nunes et $\mathrm{al}^{3}$ in his study showed that smooth borders in a focal mass were highly predictive of benign disease. Irregular and spiculated borders were more characteristic of malignant disease. Liberman and co workers ${ }^{4}$ proved in their study that for mass lesion, features that correlated with malignancy were spiculated margins, irregular shape and rim enhancement. The presence of rim enhancement was highly predictive for malignancy. Their study demonstrated a sensitivity of $96 \%$ and specificity of $79 \%$. Similar results were obtained by Boetes et $\mathrm{al}^{3}$ who showed that rim enhancement can be considered suggestive of malignancy in a mass lesion.

Kinetic curve assessment divides early enhancement (the initial rise of the enhanced curves) of mass into (2 minutes after agent injection)-slow/medium/rapid.An initial peak signal intensity within 90 seconds $>80 \%$ is defined as rapid enhancement, which is highly suggestive of malignancy.Initial peak signal intensity between $50-80 \%$ is defined as medium enhancement and $<50 \%$ is defined as slow enhancement.

The signal intensity 2 minutes after contrast injection is defined as delayed phase, which are divided into persistent/ type I- continuous increase in signal intensity,plateau/type II-signal intensity reaching peak 2 minute after contrast injection, followed by a flattening during the delayed phase),washout/type III- initial increase and subsequent decrease in signal intensity 2 minutes after contrast injection). The probability of malignancy increases from type I to type III curves. Persistent curve indicates benign and wash-out indicates malignant lesions. A plateau is considered an equivocal finding.

In our study Type III curve was 100\%specific for malignancy but overlapping was seen between the curves in benign and malignant lesions (Figure5).

Similar results were shown by Harms and colleagues ${ }^{4}$ who in their study of 74 showed significant overlap between enhancement patterns of malignant and benign lesions obtaining a sensitivity of $94 \%$ and specificity of $37 \%$. They suggested that analyzing a lesion's morphologic characteristic helps to improve the specificity in this scenario. Orel and coworkers ${ }^{4}$ evaluated both the morphologic and enhancement characteristics of suspicious breast lesions. Their data confirmed some of the previous studies that signal intensities and enhancement characteristics overlapped between benign and malignant lesions.

Few other limitations must be taken into consideration regarding the technique of kinetic curve assessment and dynamic imaging. This analysis will not be possible in malignancies which may not demonstrate rapid enhancement or may not even present as a mass;namely ductal carcinoma in situ.Similarly rapidly enhancing fibroadenomas and intramammary lymph nodes further increase the proportion of false positive reporting in kinetic analysis as proven by Gallardo and colleagues ${ }^{5}$

In our study,due to overlapping features of kinetic curves in few benign and malignant cases we predominantly relied upon morphological analysis for the categorisation of the lesion into an appropriate BIRADS category. In our study morphological analysis proved almost sufficient(95.4\%) for the correct diagnosis of lesions and kinetic analysis showed additional benefit in that it increased the level of confidence of our diagnosis.

A study done by Liberman and coworkers ${ }^{4}$ showed that for non mass like enhancement - segmental,linear,clumped, ductal enhancement were predictive of malignancy.The visually assessed kinetic parameters in this case were not significant predictors of malignancy. Nunes and coworkers ${ }^{4}$ in their study also analysed non mass like enhancement. Linear pattern was most commonly associated with ductal carcinoma in situ,while regional enhancement was not particularly predictive of either benign or malignant etiology In our study of 50 patients, 7 patients had non mass like enhancement as the primary finding on MRI breast rather than a suspicious mass. Six of them were malignancies and one benign (chronic mastitis as proven on histopathology). The malignant lesions most commonly showed focal clumped non mass like enhancement(42.8\%), followed by 
heterogenous diffuse NMLE(28.5\%) and linear NMLE (14.2\%) which coincided with suspicious calcifications on mammography and tomosynthesis (Figure 6). Multiple regional distribution (01case) was seen in the benign case (chronic mastitis on histopathology).

Microcalcifications constitute approximately $31 \%$ of lesions detected at screening mammography. Given that ductal carcinoma in situ manifests only as mammographic microcalcifications in four of five women, this is a key screening finding that warrants further diagnostic workup. ${ }^{6}$ This is in concordance with the work of Stomper and Margolin et $\mathrm{al}^{6}$ who showed that presence of microcalcifications on mammography (often referred to as early diagnosed breast cancers) is found in approximately $70 \%$ of minimal breast cancers and frequently in DCIS. Mammographically detected microcalcifications were the only sign in $72 \%$ of clinically occult DCIS lesions.

Asymmetry (according to fifth edition of ACR BIRADS mammography lexicon) represents unilateral deposits of fibroglandular tissue not conforming to the definition of a radiodense mass. The asymmetry, unlike mass, is visible on only one mammographic projection-with most such findings representing summation artifacts (a superimposition of normal breast structures). The other three types of asymmetry, although visible on more than one projection, have concaveoutward borders and usually are seen to be interspersed with fat. They can be focal (involving less than one quadrant), global(involving more than one quadrant), developing (a focal asymmetry that is new or more conspicuous over time). The latter term was added to the 2013 ACR BIRADS lexicon because this finding carries a $13.5 \%$ risk for malignancy when seen at screening mammography and $26.7 \%$ risk for malignancy when it persists at diagnostic mammography as shown by Price and colleagues ${ }^{7}$

Architectural distortion is defined as linear alterations of breast parenchyma pulled into a central focus, without a definite visible mass, resulting in radiating spiculations or thin lines pointing toward the center like a star (Ikeda et al) ${ }^{8}$ and is the third most common mammographic manifestation of non-palpable breast cancer and is the most commonly undiagnosed anomaly in mammography,being the cause of false negatives (Durand et $\mathrm{al}^{9}$ )

Architectural distortion is a classical presenting appearance for infiltrating lobular carcinoma $(16 \%$ - 20\%) and intraductal carcinoma (17\%) (Boyer and Russ et $\mathrm{al}^{10}$ )

In our study we were not able to differentiate between benign and malignant architectural distortions / asymmetries on tomosynthesis thus categorising them as BIRADS 4b. DCE- MRI proved efficacious in differentiating architectural distortion due to inflammation (multiple peripherally enhancing abscess in granulomatous mastitis, multiple peripherally enhancing fat globules in fat necrosis, multiple regional non mass like enhancement and ductal wall inflammation in chronic mastitis) or malignancy (invasion,metastasis,satellite nodules which were better visualised on DCE-MRI) (Figure-7,8,9).

This is in concordance with the study done by Bahl et al ${ }^{11}$ who showed that architectural distortion can be due to malignant lesions,such as invasive cancer or ductal carcinoma in situ or due to benign lesions like radial scar or complex sclerosing lesion,post procedural change, post surgical change, breast inflammations(either benign or malignant).Both malignant and non-malignant pathologic masses can be associated with architectural distortion. ${ }^{8}$ While the main focus of detection of architectural distortion is to identify malignancy,benign causes may manifest with a similar appearance to that of malignancy and may be mammographically indistinguishable from malignancies ${ }^{12}$

Biopsy or adjuvant USG or DCE- MRI is often necessary to exclude malignancy unless it is possible to identify an obvious benign cause, such as postsurgical or postprocedural change. ${ }^{13}$

The presence of secondary morphologic features increases suspicion of carcinoma. These features include skin and nipple changes (Figure 10), chest wall and pectoralis invasion, axillary lymphadenopathy. Architectural distortion can be present, extending beyond the limits of malignancy. Extension to the skin or fibrosis associated with the tumor can also cause dimpling and retraction. Skin thickening is seen in both benign and malignant masses but more commonly in malignant masses.Unilateral and enhancing skin thickening is suggestive of malignancy and and was seen in 3 of our cases all of which were malignant.

A hook sign symbolizes a hook like spiculated dendrite coming from the lesion's center, leading to the pectoral muscle, determined on T2-weighted images. In 4 of our cases, hook sign was present and all were malignant.

On breast MRI we detected 6 malignancies with pectoral invasion only two of which were diagnosed on tomosynthesis thus upstaging the lesion. Similar results were shown by Orel et $\mathrm{al}^{14}$, where $\mathrm{MR}$ imaging was able to contribute to local staging information for those with posterior breast tumors and proved better than tomosynthesis.

This study showed that DBT and MRI have almost equal sensitivity making DBT a good screening tool but as specificity of DCE-MRI is better it is a better diagnostic tool.

\section{Limitations}

1. The sample size was small so results can't be generalized. So study needs to be conducted on larger sample size, for reliable results.

2. The digital breast tomosynthesis and MRI were analyzed by a single observer. The observer was not blind to ultrasound or biopsy results.

3. We did not perform every MRI in second week of menstrual cycle.

4. We placed ROI manually instead of automated ROI placement by CAD.

\section{Recommendations for future study}

1. Use of a larger sample size for obtaining reliable results.

2. Additional use of DWI and CAD to improve specificity of breast MRI.

3. Imploring the efficacy of perfusion MRI, magnetic resonance spectroscopy (MRS), and magnetic resonance elastography (MRE) in improving breast MRI specificity

4. Using higher field strength 7.0 Tesla imaging to obtain higher resolution of breast MRI 
5. Use of 18 channel closely fitted coil for bidirectional parallel imaging of the breast.

6. Use of abbreviated breast MRI protocol to obtain balance between shorter scan time and appropriate imaging.

7. Further advancements in mammography in the form of contrast enhanced mammography may further improve the evaluation of breast lesions and may take it at par to breast MRI.

\section{CONCLUSION}

Breast malignancy has become a global epidemic today. The only key to successfully deal with this is by early diagnosis and prompt management of the patient. A tremendous efficacy in detecting micro-calcifications (often the earliest sign of malignancy) makes mammography the standard investigation for routine screening for breast malignancy. It has few limitations due to superimposition of breast tissue but recent advancement in the form of tomosynthesis has removed much of the limitations of two-dimensional imaging. The main problem with DBT is its lesser availability due to its high cost.

Mammography and DBT are very efficient in assessing mass, microcalcification using ACR BIRADS lexicon except in cases of global asymmetry for which additional second look ultrasound or dynamic contrast enhanced MRI can be used for evaluation. MRI offers superb visualization of posterior breast tissue, axillary lymph node involvement, multicentric/ multifocal/ contralateral breast involvement, invasion or metastasis. Morphological analysis of DCE-MRI triumphs over kinetic analysis of time signal intensity curves in determining whether the lesion is of benign or malignant etiology. Ideally both morphologic and kinetic studies should be in concordance to each other but in case of discrepancy between the two, the more suspicious finding must be given importance with BI-RADS categorization being based on that

\section{REFERENCES}

1. Shreshtha Malvia, Sarangadhara Appalaraju Bagadi, Uma S. Dubey, Sunita Saxena. Epidemiology of breast cancer in Indian women. Asia Pac J Clin Oncol. 2017;13(4):289-295

2. Global surveillance of trends in cancer survival 200014 (CONCORD -3): Analysis of individual records for 37513025 patients diagnosed with one of 18 cancers from 322 population - based registries in 71 countries; Claudia Allemani, Tomohir Matsuda, Veronica Di Carlo et al; January 30, 2018

3. Mitsuhiro Tozaki. Interpretation of breast MRI: correlation of kinetic and morphological parameters with pathological findings; Magnetic Resonance in Medical Sciences 2004;3(2):189-197,

4. Elizabeth A. Morris, Laura Liberman et al. Breast MRI diagnosis and intervention book, chapter 1 (p-3-6), January 2005

5. Gallardo X, Sentis M, Castaner E et al. Enhancement of intramammary lymph nodes with lymphoid hyperplasia. a potential pitfall in breast MRI. Eur Radiol. 1998; 8(4): 1662-1665

6. Barbara Bennani-Baiti, Pascal A. Baltzer. MR Imaging for Diagnosis of Malignancy in Mammographic Microcalcifications: A Systematic Review and MetaAnalysis Radiology. 2017;283(3):692-701.

7. Price ER, Joe BN, Sickles EA. The developing asymmetry: revisiting a perceptual and diagnostic challenge. Radiology 2015;274(3):642-651.

8. Mayson. M. Wansi, Caroline Edward, Ayad Doha Abdo Mohammed: Evaluation of Breast Architecture and Mass Morphology in Digital Mammography using BIRADS. IOSR Journal of Dental and Medical Sciences (IOSR-JDMS) 2019;18(4):75-79.

9. Durand, M. A. et al. Tomosynthesis-detected architectural distortion: management algorithm with radiologic-pathologic correlation Radiographics. Radiological Society of North America 2016;36(3):311321.

10. Boyer, B. and Russ, E. Anatomical-radiological correlations. Architectural distortions Diagnostic and Interventional Imaging. Elsevier Masson SAS 2014;95(3):134-140.

11. Bahl, M. et al. Architectural Distortion on Mammography: Correlation with Pathologic Outcomes and Predictors of Malignancy. American Journal of Roentgenology, 2015;205(1):1339-1345

12. Melissa A. Durand, Steven Wang, Regina J. Hooley, Madhavi Raghu, Liane E. Philpotts. Tomosynthesisdetected Architectural Distortion: Management Algorithm with Radiologic-Pathologic Correlation RadioGraphics 2016; 36(6):311-321.

13. Shantanu Gaur, Priscilla J. Slanetz, Ronald L. Eisenberg: Architectural Distortion of the Breast. AJR 2013; 201:W662-W670 0361-803X/13/2015-W662.

14. Orel SG, Schnall, Powell CM et al. Staging of suspected breast cancer. Effect of MR imaging and MR guided biopsy. Radiology 1995; 196(5):115-122.21.

Source of Support: Nil; Conflict of Interest: None

Submitted: 10-05-2019; Accepted: 01-06-2019; Published online: 15-06-2019 